Article

\title{
Associations between SNPs in Intestinal Cholesterol Absorption and Endogenous Cholesterol Synthesis Genes with Cholesterol Metabolism
}

\author{
Maite M. Schroor ${ }^{*}+{ }^{+}$, Fatma B. A. Mokhtar ${ }^{*}+$, Jogchum Plat $\mathbb{1}$ and Ronald P. Mensink \\ Department of Nutrition and Movement Sciences, NUTRIM School of Nutrition and Translational Research in \\ Metabolism, Maastricht University, 6200 MD Maastricht, The Netherlands; j.plat@maastrichtuniversity.nl (J.P.); \\ r.mensink@maastrichtuniversity.nl (R.P.M.) \\ * Correspondence: maite.schroor@maastrichtuniversity.nl (M.M.S.); \\ fatma.mokhtar@maastrichtuniversity.nl (F.B.A.M.); Tel.: +31-(0)43-3884258 (M.M.S.); \\ +31-(0)43-3881313 (F.B.A.M.) \\ + These authors contributed equally to this work.
}

check for

updates

Citation: Schroor, M.M.; Mokhtar, F.B.A.; Plat, J.; Mensink, R.P.

Associations between SNPs in

Intestinal Cholesterol Absorption and Endogenous Cholesterol Synthesis Genes with Cholesterol Metabolism. Biomedicines 2021, 9, 1475 .

https://doi.org/10.3390/

biomedicines 9101475

Academic Editor: Julie Chan

Received: 26 August 2021

Accepted: 11 October 2021

Published: 14 October 2021

Publisher's Note: MDPI stays neutral with regard to jurisdictional claims in published maps and institutional affiliations.

Copyright: (c) 2021 by the authors. Licensee MDPI, Basel, Switzerland. This article is an open access article distributed under the terms and conditions of the Creative Commons Attribution (CC BY) license (https:/ / creativecommons.org/licenses/by/ $4.0 /)$.
Abstract: Single nucleotide polymorphisms (SNPs) have been associated with cholesterol metabolism and may partly explain large inter-individual variability in intestinal cholesterol absorption and endogenous cholesterol synthesis rates. This cross-sectional study therefore examined whether SNPs in genes encoding for proteins involved in intestinal cholesterol absorption $(A B C G 5, A B C G 8$, and NPC1L1) and endogenous cholesterol synthesis (CYP51A1, DHCR7, DHCR24, HMGCR, HSD17B7, $L B R$, and MSMO1) were associated with intestinal cholesterol absorption markers (total cholesterol (TC) standardized campesterol and sitosterol levels), an endogenous cholesterol synthesis marker (TC-standardized lathosterol levels), and serum low-density lipoprotein cholesterol (LDLC) concentrations in a European cohort. ABCG5 (rs4245786) and the tag SNP ABCG8 (rs4245791) were significantly associated with serum campesterol and/or sitosterol levels. In contrast, NPC1L1 (rs217429 and rs217416) were significantly associated with serum lathosterol levels. The tag SNP in HMGCR (rs12916) and a SNP in LBR (rs12141732) were significantly associated with serum LDL-C concentrations. SNPs in the cholesterol absorption genes were not associated with serum LDL-C concentrations. SNPs in CYP51A1, DHCR24, HSD17B7, and MSMO1 were not associated with the serum non-cholesterol sterols and LDL-C concentrations. Given the variable efficiency of cholesterollowering interventions, the identification of SNPs associated with cholesterol metabolism could be a step forward towards personalized approaches.

Keywords: genetic variants; genetics; cholesterol metabolism; cholesterol absorption; cholesterol biosynthesis; sterols; campesterol; sitosterol; lathosterol

\section{Introduction}

Cholesterol homeostasis is determined by the interaction between various complex processes including intestinal dietary and biliary cholesterol absorption, and endogenous cholesterol synthesis [1,2]. For the uptake of sterols into the enterocyte, the apical transporter Niemann-Pick C1-Like 1 (NPC1L1) plays a key role [3]. After absorption, the sterol efflux pump ATP-binding cassette (ABC) transporters G5 and G8 secrete a fraction of these sterols back into the intestinal lumen, while the remaining part is incorporated into chylomicrons and secreted into the circulation (Figure S1) [4]. De novo cholesterol synthesis, which involves approximately 30 reactions and more than 20 different enzymes, mainly takes place in the liver [2]. Other tissues, however, synthesize cholesterol as well [2]. The endogenous cholesterol synthesis pathway starts with acetyl-CoA, which is converted into the intermediate lanosterol in a multistep process. Lanosterol is ultimately converted into cholesterol via either the Bloch or the Kandutsch-Russell pathway (Figure S2). The intermediates in these two pathways differ, but the same enzymes are involved [5-8]. To 
estimate fractional intestinal cholesterol absorption, cholesterol-standardized campesterol and sitosterol levels can be used, while those of the Kandutsch-Russell pathway intermediate lathosterol reflect endogenous cholesterol synthesis rates. The use of these markers has been validated by correlating their plasma levels to stable isotope tracer measurements [9].

A reciprocal relation exists between intestinal cholesterol absorption and endogenous cholesterol synthesis [10]. For example, statin treatment decreases cholesterol synthesis but increases cholesterol absorption [11], while ezetimibe treatment results in the opposite effects [12]. Furthermore, large inter-individual differences are present in relative intestinal cholesterol absorption and endogenous cholesterol synthesis rates. To illustrate, intestinal cholesterol absorption values ranged from approximately $29 \%$ to $80 \%$ in healthy adults. However, within subject-variability was small [13]. For the cholesterol synthesis marker lathosterol, an intra-individual variation of around $23 \%$ and an inter-individual variation of more than $50 \%$ has been reported for healthy adults [14]. Genetic variants, including singlenucleotide polymorphisms (SNPs), might at least partly explain these large inter-individual variations and the wide ranges between individuals in responses to lipid-lowering medications [15]. In fact, some SNPs in intestinal cholesterol absorption genes have already been associated with fractional cholesterol absorption rates [16-19]. Additionally, several studies have reported associations between SNPs in genes related to intestinal cholesterol absorption and endogenous cholesterol synthesis with lipid-lowering effects of both pharmacological [20-23] and dietary interventions [24,25]. However, whether these associations relate to differences in intestinal cholesterol absorption and endogenous cholesterol synthesis rates has unfortunately not been documented. Identification of SNPs associated with intestinal cholesterol absorption and endogenous cholesterol synthesis is important, as findings may contribute to the development of personalized interventions aimed at improving cholesterol metabolism. The present study therefore investigated in a European population the relation between a number of selected SNPs in genes essential in intestinal cholesterol absorption-ABCG5, ABCG8, and NPC1L1-and SNPs in genes involved in endogenous cholesterol synthesis-CYP51A1, DHCR7, DHCR24, HMGCR, HSD17B7, LBR, and $M S M O 1$ - with serum intestinal cholesterol absorption markers (total cholesterol (TC) standardized levels of campesterol and sitosterol), an endogenous cholesterol synthesis marker (TC-standardized levels of lathosterol), and LDL-C concentrations.

\section{Materials and Methods}

\subsection{Study Population}

The present study included participants' baseline data from five human intervention studies (Study 1 to Study 5), performed between 1997 and 2012 at Maastricht University, the Netherlands. All participants were recruited from Maastricht and the surrounding area, and data from $\mathrm{N}=456$ were available for the present study. Overall, the study sample consisted of healthy adults aged $\geq 18$ years old. The body mass index (BMI) was calculated for each participant by diving their body weight $(\mathrm{kg})$ by the square of height $(\mathrm{m})$. Most participants had a normal weight $(\mathrm{N}=225 ; 49.3 \%)$ or were overweight $(\mathrm{N}=179 ; 39.3 \%)$. BMI of few participants fell within the underweight $(\mathrm{N}=7 ; 1.5 \%)$, obesity class $\mathrm{I}(\mathrm{N}=28$, $6.1 \%)$ or obesity class II $(\mathrm{N}=6 ; 1.3 \%)$ range [26]. None of the participants used medication known to affect lipid metabolism. Details of the studies have been published [27-30], except for Study 4, which was a 6-week randomized, double-blinded, placebo-controlled parallel trial evaluating effects of plant-sterol ester supplementation as part of a combined lifestyle intervention. For the analysis of this project, we only used samples that were collected at baseline or at the end of a control period. All studies were approved by the Medical Ethics Committee of Maastricht University and were conducted according to the principles laid down in the Declaration of Helsinki. Written informed consent was obtained from all participants. 


\subsection{Blood Sampling and Biochemical Measurements}

Blood samples were drawn from participants after an overnight fast. At least one hour after venipuncture, serum was obtained by centrifugation at $2000 \times \mathrm{g}$ for $15-30 \mathrm{~min}$ at $4{ }^{\circ} \mathrm{C}$ and aliquots were stored at $-80^{\circ} \mathrm{C}$. The concentrations of TC (CHOD/PAP method; Roche Diagnostics Systems Hoffmann-La Roche Ltd., Basel, Switzerland), high-density lipoprotein cholesterol (HDL-C) (precipitation method by adding phosphotungstic acid and magnesium ions, and CHOD/PAP method; Roche Diagnostics Systems HoffmannLa Roche Ltd., Basel, Switzerland) and triacylglycerol (TAG) corrected for free glycerol (GPO-Trinder; Sigma Diagnostics, St Louis, USA) were determined in serum by using enzyme-based methods. LDL-C concentrations were calculated using the Friedewald equation [31].

Serum concentrations of the intestinal cholesterol absorption markers campesterol and sitosterol, and the endogenous cholesterol synthesis marker lathosterol were analyzed using gas chromatography with flame-ionization detection (GC-FID) in Study 1 and Study 5, while GC-mass spectrometry (GC-MS) was used in the three other studies. Further details on the non-cholesterol sterol analysis have been presented in the article by Mackay et al. [32]. Campesterol, sitosterol, and lathosterol concentrations are transported in plasma by cholesterol-rich lipoproteins, and therefore their concentrations were corrected for the differing number of lipoprotein particles by standardizing the concentrations of the markers to the TC concentrations $\left(10^{2} \times \mu \mathrm{mol} / \mathrm{mmol} \mathrm{TC}\right)$ as measured with the CHOD/PAP method.

\subsection{DNA Extraction, Genotyping, and Quality Control}

Genomic DNA was isolated from either full blood or buffy coats using the QIAamp genomic DNA isolation kit (Westburg BV, Leusden, the Netherlands) according to the instructions of the manufacturer. After isolation, the purity of the genomic DNA was checked by measuring the $260 / 280 \mathrm{~nm}$ and the $260 / 230 \mathrm{~nm}$ ratios (NanoDrop; ND-1000 spectrophotometer, Isogen Lifescience B.V., De Meern, The Netherlands). For all samples, ratios varied between 1.7 and 1.9 and around 2.0, respectively. DNA concentrations were calculated using the relationship that an $\mathrm{A}_{260}$ of 1.0 corresponds with $50 \mu \mathrm{g} / \mathrm{mL}$ DNA. All samples were stored at $-80{ }^{\circ} \mathrm{C}$ after isolation. After thawing, the quality of about $5 \%$ of the samples was tested by evaluating the degradation of DNA on agarose gels before further analysis. Results indicated that the quality of these samples was sufficient for genotyping. In the end, 471 DNA samples were genotyped by using the Axiom ${ }^{\mathrm{TM}}$ Precision Medicine Research Array (PMRA) Kit (Thermo Fisher Scientific, Waltham, MA, USA) [33].

After running the arrays, the software package PLINK (version 1.90 beta; www.coggenomics.org/plink/1.9/) [34] was used to exclude SNPs: (1) with $>2 \%$ missing data, (2) located on sex chromosomes, (3) with a minor allele frequency (MAF) $<0.05$, or (4) that deviated from Hardy-Weinberg Equilibrium (HWE) based on a $p$-value $<1 \times 10^{-10}$. Six subjects were removed, because they had a heterozygosity rate \pm 3 standard deviations (SDs) from the mean heterozygosity rate. Nine subjects were excluded because there was a sex discrepancy between DNA results with clinical records. Ultimately, 456 samples and 306,898 SNPs passed the quality-control criteria. Only SNPs in genes with a clear role in intestinal cholesterol absorption (ABCG5, ABCG8, and NPC1L1) or endogenous cholesterol synthesis (CYP51A1, DHCR7, DHCR24, HMGCR, HSD17B7, LBR, and MSMO1) that were present on the array and had passed the quality control steps were included in this study. An overview of the full gene names is provided in Table S1. The rs-numbers of the selected SNPs are presented, except for two SNPs in ABCG8 for which the rs-numbers were unknown. For these SNPs, their Affymetrix SNP ID (AX-number), i.e., their unique probe set identifier, is given. Table S2 presents information about these two SNPs that was provided by the PMRA array. 


\subsection{Statistics}

Continuous values are reported as mean \pm SD and categorical values as $\mathrm{N}(\%)$. Visual inspection of histograms and Q-Q plots of the residuals showed a skewed distribution for TAG and concentrations were therefore log-transformed. Analysis of variance (ANOVA) was used to examine whether continuous variables differed significantly between the five studies. A chi-square test was used for categorical variables.

Possible deviations of the genotype frequencies from those expected under HardyWeinberg equilibrium (HWE) were assessed using chi-square tests in Microsoft Excel. Thereafter, SNPs with a genotype group with a frequency of $<12$ participants, which equals $<2.5 \%$ of the sample size, were moved to the supplements. All SNPs in DHCR7 were moved to the supplements due to this reason. Only for SNPs with a genotype group with a frequency of $>12$ participants, linkage disequilibrium (LD) was estimated and reported as $\mathrm{r}^{2}$-values for pairs of SNPs $<500 \mathrm{kB}$ apart using the Haploview software package (version 4.1, Broad Institute of MIT and Harvard, Cambridge, MA, USA) [35]. A threshold of $\mathrm{r}^{2} \geq 0.8$ was used to define SNPs in LD. Haplotype blocks were constructed in Haploview by using the default algorithm as defined by Gabriel et al. [36]. In short, blocks were generated by this algorithm when at least $95 \%$ of the informative SNPs were in strong LD [36]. Furthermore, the Tagger program in Haploview version 4.1 was used to select tag SNPs using the pairwise tagging approach [35]. Selection criteria were a $\mathrm{r}^{2}$ threshold $\geq 0.8$ and a log of the likelihood odds ratio (LOD) threshold of 3.0. Results of the statistical analysis of the tag SNPs are presented in the main text, whereas results for the captured SNPs have been placed in the supplemental information.

Linear regression analyses, corrected for the factor study, were used to examine associations among the TC-standardized non-cholesterol sterols and LDL-C concentrations. Additionally, the general linear model (GLM) was used to examine associations between the SNPs with serum non-cholesterol sterol levels, and LDL-C and TC concentrations. The analyses were adjusted for the factor study. In case of a statistically significant effect of a SNP, the differences in TC-standardized non-cholesterol sterol levels, serum LDL-C concentrations, or serum TC concentrations between the genotype groups were compared with a Bonferroni post-hoc test. The Benjamini-Hochberg multiple testing correction with a false discovery rate of 0.2 was applied to the GLM results for each gene separately. Only SNPs with genotype groups consisting of at least 12 individuals were included in the Benjamini-Hochberg correction. If the original $p$-value obtained from the general linear model analysis was smaller than the Benjamini-Hochberg critical value, the $p$-value was considered statistically significant. Next, for SNPs that were significantly associated with TC-standardized non-cholesterol sterols or LDL-C concentrations, an additive, dominant, or recessive multiple linear regression model was built with adjustment for the factor study. The additive model was used when the Bonferroni post-hoc test indicated that all three genotypes were significantly different or when the post-hoc test did not show which genotypes differed significantly. A dominant or recessive model was used when the Bonferroni post-hoc indicated a significant difference between only two genotypes. A dominant model was used if the least frequent homozygous genotype (e.g., aa) and the heterozygous genotype (e.g., aA) had a comparable relation with the outcome (i.e., the non-cholesterol sterols or LDL-C). The dominant model used the major homozygous group as reference, hence, AA was compared with aa + aA. Moreover, a recessive model was used if the least frequent homozygous genotype and the heterozygous genotype did not have a comparable relation with the outcome. The recessive model thus compared AA + aA with aa. All analyses were carried out using SPSS for Mac OS X (version 26.0, SPSS Inc., Chicago, IL, USA).

\section{Results}

Baseline characteristics for all participants and the five studies separately are shown in Table S3. Significant differences between the studies were reported for all characteristics of the participants (all $p<0.05)$, except for gender $(p=0.064)$. 
3.1. Associations between Markers for Cholesterol Absorption and Cholesterol Synthesis, and Serum LDL-C Concentrations

Linear regression analyses showed that, after controlling for the factor study, sitosterol was positively associated with campesterol $\left(\beta=1.39 \times 10^{2} \mu \mathrm{mol} / \mathrm{mmol} \mathrm{TC} ; p<0.001\right)$ and inversely with lathosterol $\left(\beta=-0.09 \times 10^{2} \mu \mathrm{mol} / \mathrm{mmol} \mathrm{TC} ; p=0.025\right)$. In addition, campesterol showed a significant inverse association with lathosterol $\left(\beta=-0.10 \times 10^{2} \mu \mathrm{mol} / \mathrm{mmol} \mathrm{TC}\right.$; $p<0.001)$. Campesterol, sitosterol, and lathosterol were not significantly associated with serum LDL-C concentrations (all $p>0.05$ ) (Table S4).

\subsection{The Location and Allele Frequencies of the Selected SNPS}

Table S5 shows the location and allele frequencies of the selected SNPs. The majority of SNPs were located in an intron and all SNPs had a call rate of $\geq 98.2 \%$. The reference and alternative allele frequencies of the SNPs in our cohort were comparable to those of the European population, which were obtained from the National Center for Biotechnology Information (NCBI) [37]. Five of the 12 selected SNPs in the ABCG8 gene (AX_11180448, rs41360247, rs4245791, rs4299376, rs6544713) deviated significantly from HWE $(p<0.05)$. All other SNPs were in HWE (all $p>0.05$ ).

\subsection{Linkage Disequilibrium and Tagging for SNPs in Genes Related to Intestinal Cholesterol Absorption}

SNPs in ABCG8 (rs4299376, rs6544713, and rs4245791) were in high LD (all r² >0.90) and consequently included in a haplotype block (Figure 1a). Haplotype block 2 included ABCG8 (rs13390041, rs4077440, and rs3795860). Of these SNPs, rs13390041 and rs3795860 showed a high LD $\left(\mathrm{r}^{2}=0.98\right)$. The tag SNP ABCG8 (rs4245791) captured rs6544713 and rs4299376, while tag SNP ABCG8 (rs3795860) captured rs13390041 (Table 1). For SNPs in $A B C G 5$ (Figure S3a) and NPC1L1 (Figure S3b), no high LD was found $\left(\right.$ all $\left.\mathrm{r}^{2}<0.70\right)$.

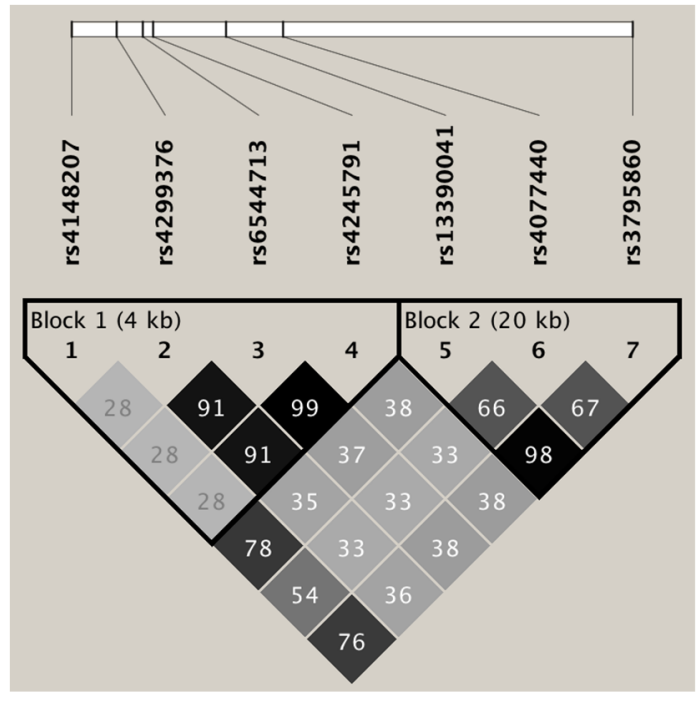

(a)

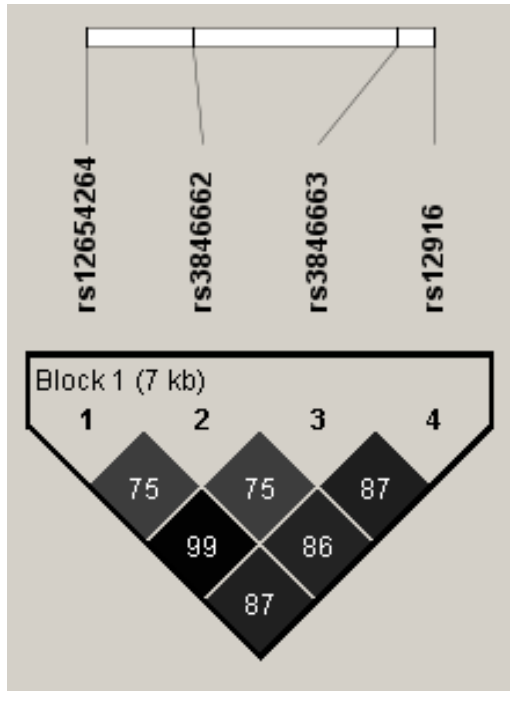

(b)

Figure 1. Pairwise LD among (a) 7 SNPs in ABCG8 and (b) 4 SNPs in HMGCR is indicated in the diamond shapes. The triangles mark the two haplotype blocks within this region (based on the confidence interval of $\mathrm{D}^{\prime}$ ). The shading with a dark grey to white gradient indicates the level of higher to lower LD between each pair of SNPs based on the $\mathrm{r}^{2}$-value. The LD plot was created by Haploview version 4.1 [35].

\subsection{Linkage Disequilibrium and Tagging for SNPs in Genes Related to Endogenous Cholesterol Synthesis}

All SNPs in HMGCR were in (borderline) LD (all $\mathrm{r}^{2} \geq 0.75$ ) and consequently all SNPs were included in one single haplotype block (Figure 1b). One tag SNP in HMGCR was selected (rs12916), which captured rs12654264, rs3846662, and rs3846663 (Table 1). For 
DHCR24, rs6676774 and rs7551288 were in high LD $\left(\mathrm{r}^{2}=0.90\right)$ and DHCR24 (rs6676774) was selected as a tag SNP for rs7551288 (Figure S4c; Table 1). None of the other SNPs in DHCR24, as well as the SNPs in LBR were in pairwise LD (all $\mathrm{r}^{2}<0.80$ ) (Figure S4).

Table 1. Tag SNPs and their captured SNPs with their corresponding $\mathrm{r}^{2}$-values.

\begin{tabular}{cccc}
\hline Gene & Tag SNP & Captured SNP & R $^{\mathbf{2}}$-Value \\
\hline ABCG8 & rs4245791 & rs6544713 & 0.995 \\
& rs4245791 & rs4299376 & 0.919 \\
& rs3795860 & rs13390041 & 0.982 \\
DHCR24 & rs6676774 & rs7551288 & 0.906 \\
HMGCR & rs12916 & rs12654264 & 0.872 \\
& rs12916 & rs3846662 & 0.862 \\
& rs12916 & rs3846663 & 0.879 \\
\hline
\end{tabular}

Tag SNPs and their captured SNPs were selected using the Tagger program within Haploview version 4.1. [35].

3.5. Associations between SNPs in ABCG5, ABCG8, and NPC1L1 with TC-Standardized Serum Non-Cholesterol Sterol Levels and Serum LDL-C Concentrations

Significant associations were found for a SNP in ABCG8 (rs4245791; $p<0.001$ ) with both TC-standardized serum campesterol and TC-standardized serum sitosterol levels. ABCG5 (rs4245786) was also significantly associated with TC-standardized sitosterol levels $(p=0.041)$. In addition, two SNPs in NPC1L1 (rs217429 and rs217416) were significantly related with TC-standardized serum lathosterol levels $(p<0.05)$ (Table 2). After BenjaminiHochberg multiple testing correction, all associations remained significant. Results for SNPs with a genotype group $<12$ participants are presented in Table S6. A recessive model was built for NPC1L1 (rs217429 and rs217416) with lathosterol levels (Figure S5). The additive models for ABCG5 (rs4245786) with sitosterol, and for ABCG8 (rs4245791) with sitosterol and campesterol levels can be found in Table S7. No significant associations were observed between SNPs in ABCG5, ABCG8, or NPC1L1 with serum LDL-C concentrations (all $p>0.05$ ) (Table 2) or TC concentrations (all $p>0.05$ ) (Table S8).

3.6. Associations between SNPs in CYP51A1, DHCR24, HMGCR, HSD17B7, LBR, and MSMO1 with TC-Standardized Serum Non-Cholesterol Sterol Levels and Serum LDL-C Concentrations

None of the SNPs in genes essential in endogenous cholesterol synthesis showed a significant association with TC-standardized campesterol, sitosterol or lathosterol serum levels (all $p>0.05)$. Significant associations were reported for HMGCR (rs12916) and LBR (rs12141732) with serum LDL-C concentrations (all $p<0.05$ ) (Table 3). Dominant models for these SNPs can be found in Figure S6. SNPs in CYP51A1, DHCR24, HSD17B7, and MSMO1 were not significantly associated with serum LDL-C concentrations (all $p>0.05$ ). Table S9 presents associations for SNPs with a genotype group $<12$ participants. Results for serum TC concentrations (Table S10) are comparable to these of serum LDL-C concentrations (Table 3). 


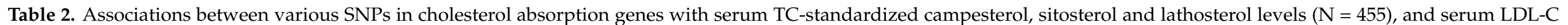
concentrations $(\mathrm{N}=456)$.

\begin{tabular}{|c|c|c|c|c|c|c|c|c|c|c|c|c|}
\hline \multirow[t]{2}{*}{ Gene } & \multirow[t]{2}{*}{ SNP } & \multirow[t]{2}{*}{ Genotype } & \multirow[t]{2}{*}{$\mathbf{N}$} & \multicolumn{2}{|c|}{$\begin{array}{c}\text { Campesterol } \\
10^{2} \times \mu \mathrm{mol} / \mathrm{mmol} \mathrm{TC}\end{array}$} & \multicolumn{2}{|c|}{$\begin{array}{c}\text { Sitosterol } \\
10^{2} \times \mu \mathrm{mol} / \mathrm{mmol} \mathrm{TC}\end{array}$} & \multicolumn{2}{|c|}{$\begin{array}{c}\text { Lathosterol } \\
10^{2} \times \mu \mathrm{mol} / \mathrm{mmol} \mathrm{TC}\end{array}$} & \multirow[t]{2}{*}{$\mathbf{N}$} & \multicolumn{2}{|c|}{$\begin{array}{l}\text { LDL-C } \\
\mathrm{mmol} / \mathrm{L}\end{array}$} \\
\hline & & & & Mean $(95 \% \mathrm{CI})$ & $p$-Value & Mean $(95 \%$ CI) & $p$-Value & Mean $(95 \% \mathrm{CI})$ & $p$-Value & & Mean $(95 \%$ CI) & $p$-Value \\
\hline \multirow[t]{11}{*}{$A B C G 5$} & rs4245786 & AA & 266 & $252(236-267)$ & & $152(142-162)$ & & $120(112-129)$ & & 266 & $3.44(3.30-3.57)$ & \\
\hline & & AG & 160 & $230(212-249)$ & 0.074 & $136(124-148)$ & $0.041^{\$}$ & $120(110-131)$ & 0.959 & 161 & $3.34(3.18-3.50)$ & 0.306 \\
\hline & & GG & 29 & $259(222-296)$ & & $154(130-178)$ & & $123(103-144)$ & & 29 & $3.23(2.90-3.55)$ & \\
\hline & rs7599296 & AA & 15 & $261(210-312)$ & & 164 (131-197) & & $109(81-137)$ & & 15 & $3.40(2.95-3.85)$ & \\
\hline & & AG & 141 & $255(236-274)$ & 0.228 & $152(140-165)$ & 0.173 & 119 (108-130) & 0.653 & 141 & $3.38(3.21-3.54)$ & 0.980 \\
\hline & & GG & 299 & $239(224-254)$ & & 143 (133-152) & & $122(113-130)$ & & 300 & $3.39(3.26-3.52)$ & \\
\hline & & $\mathrm{TC}$ & 219 & $251(235-268)$ & 0.297 & 148 (137-159) & 0.803 & $117(108-126)$ & 0.217 & 219 & $3.42(3.28-2.57)$ & 0.561 \\
\hline & & $\mathrm{CC}$ & 161 & $242(223-260)$ & & $146(134-158)$ & & $126(116-137)$ & & 162 & $3.39(3.23-3.55)$ & \\
\hline & rs13396273 & $\mathrm{TT}$ & 53 & $236(207-264)$ & & $144(126-163)$ & & $116(101-132)$ & & 53 & $3.36(3.11-3.60)$ & \\
\hline & & TC & 214 & $251(234-267)$ & 0.431 & 148 (138-159) & 0.819 & 119 (109-128) & 0.526 & 214 & $3.40(3.26-3.55)$ & 0.922 \\
\hline & & $\mathrm{CC}$ & 188 & $240(222-257)$ & & $145(133-156)$ & & $124(114-134)$ & & 189 & $3.38(3.22-3.53)$ & \\
\hline \multirow[t]{13}{*}{ ABCG8 } & rs4148207 & TT & 156 & $249(231-268)$ & & 151 (139-163) & & $121(111-131)$ & & 157 & $3.34(3.18-3.50)$ & \\
\hline & & $\mathrm{TC}$ & 227 & $243(226-259)$ & 0.757 & 145 (123-155) & 0.364 & $121(112-130)$ & 0.713 & 227 & $3.43(3.29-3.58)$ & 0.530 \\
\hline & & $\mathrm{CC}$ & 72 & $241(216-266)$ & & 139 (123-155) & & $116(102-129)$ & & 72 & $3.35(3.13-3.57)$ & \\
\hline & & $\mathrm{CC}$ & 94 & $234(211-257)$ & & $138(123-152)$ & & $115(102-127)$ & & 94 & $3.29(3.09-3.49)$ & \\
\hline & rs4077440 & $\mathrm{TT}$ & 92 & $256(233-279)$ & & $154(140-169)$ & & $120(107-132)$ & & 92 & $3.38(3.18-3.58)$ & \\
\hline & & $\mathrm{TC}$ & 217 & $249(232-266)$ & 0.129 & 149 (138-159) & 0.125 & $124(115-133)$ & 0.378 & 218 & $3.45(3.31-1.60)$ & 0.252 \\
\hline & & $\mathrm{CC}$ & 145 & $232(213-251)$ & & $138(126-150)$ & & $116(105-126)$ & & 145 & $3.30(3.13-3.46)$ & \\
\hline & AX_82902928 & - & 197 & $248(231-265)$ & & $151(140-161)$ & & $120(111-130)$ & & 197 & $3.40(3.25-3.55)$ & \\
\hline & & $-A C$ & 192 & $240(223-258)$ & 0.752 & $141(130-165)$ & 0.334 & $120(110-130)$ & 0.955 & 193 & $3.43(3.28-3.58)$ & 0.145 \\
\hline & & ACAC & 66 & $246(219-272)$ & & 147 (130-165) & & $122(108-137)$ & & 66 & $3.19(2.60-3.42)$ & \\
\hline & rs4245791 + & TT & 206 & $221(205-237) \mathrm{A}$ & & $130(120-141)^{\mathrm{A}}$ & & $123(114-132)$ & & 206 & $3.32(3.17-3.47)$ & \\
\hline & & $\mathrm{TC}$ & 215 & $256(239-272)^{\text {В }}$ & $<0.001^{\$}$ & $153(143-164)^{\text {В }}$ & $<0.001^{\$}$ & $119(109-128)$ & 0.642 & 216 & $3.46(3.31-3.61)$ & 0.239 \\
\hline & & $\mathrm{CC}$ & 34 & $315(282-349) \mathrm{C}$ & & $180(176-219)^{\mathrm{C}}$ & & $117(97-136)$ & & 34 & $3.34(3.04-3.65)$ & \\
\hline
\end{tabular}


Table 2. Cont.

\begin{tabular}{|c|c|c|c|c|c|c|c|c|c|c|c|c|}
\hline \multirow[t]{2}{*}{ Gene } & \multirow[t]{2}{*}{ SNP } & \multirow[t]{2}{*}{ Genotype } & \multirow[t]{2}{*}{$\mathbf{N}$} & \multicolumn{2}{|c|}{$\begin{array}{c}\text { Campesterol } \\
10^{2} \times \mu \mathrm{mol} / \mathrm{mmol} \mathrm{TC}\end{array}$} & \multicolumn{2}{|c|}{$\begin{array}{ll} & \text { Sitosterol } \\
10^{2} \times \mu \mathrm{mol} / \mathrm{mmol} \mathrm{TC}\end{array}$} & \multicolumn{2}{|c|}{$\begin{array}{c}\text { Lathosterol } \\
10^{2} \times \mu \mathrm{mol} / \mathrm{mmol} \mathrm{TC}\end{array}$} & \multirow[t]{2}{*}{$\mathbf{N}$} & \multicolumn{2}{|c|}{$\begin{array}{c}\text { LDL-C } \\
\mathrm{mmol} / \mathrm{L}\end{array}$} \\
\hline & & & & Mean $(95 \% \mathrm{CI})$ & $p$-Value & Mean $(95 \% \mathrm{CI})$ & $p$-Value & Mean $(95 \% \mathrm{CI})$ & $p$-Value & & Mean $(95 \%$ CI $)$ & $p$-Value \\
\hline \multirow[t]{12}{*}{ NPC1L1 } & rs217429 & $\mathrm{AA}$ & 259 & $239(223-254)$ & & $142(132-152)$ & & $119(110-128)^{\mathrm{A}}$ & & 259 & $3.37(3.23-3.50)$ & \\
\hline & & $\mathrm{AC}$ & 169 & $256(238-275)$ & 0.190 & $154(142-166)$ & 0.134 & $117(107-127) \mathrm{A}$ & $0.017^{\#}$ & 170 & $3.42(3.26-3.58)$ & 0.825 \\
\hline & & $\mathrm{CC}$ & 27 & $238(200-276)$ & & $146(121-170)$ & & $149(128-170)^{\text {В }}$ & & 27 & $3.39(3.06-3.73)$ & \\
\hline & rs217416 & $\mathrm{TT}$ & 239 & $240(223-256)$ & & $143(132-153)$ & & $119(110-127) \mathrm{A}$ & & 239 & $3.40(3.26-3.54)$ & \\
\hline & & $\mathrm{TC}$ & 189 & $254(237-272)$ & 0.208 & $153(141-164)$ & 0.236 & $118(108-128)^{\mathrm{A}}$ & $0.020^{\#}$ & 190 & $3.38(3.23-3.54)$ & 0.922 \\
\hline & & $\mathrm{CC}$ & 25 & $228(188-267)$ & & $140(114-165)$ & & $149(128-171)^{\text {В }}$ & & 25 & $3.33(2.98-3.67)$ & \\
\hline & rs11763759 & $\mathrm{TT}$ & 208 & $244(227-261)$ & & $145(134-156)$ & & $120(111-130)$ & & 209 & $3.42(3.27-3.56)$ & \\
\hline & & $\mathrm{TC}$ & 202 & $246(229-263)$ & 0.961 & 147 (136-158) & 0.938 & $120(111-129)$ & 0.953 & 202 & $3.31(3.16-3.46)$ & 0.084 \\
\hline & & $\mathrm{CC}$ & 43 & $242(211-273)$ & & 149 (128-169) & & $123(106-140)$ & & 43 & $3.62(3.35-3.89)$ & \\
\hline & rs2072183 & $\mathrm{CC}$ & 18 & $260(213-307)$ & & $154(123-184)$ & & $121(95-147)$ & & 18 & $3.33(2.91-3.75)$ & \\
\hline & & CG & 173 & $254(235-272)$ & 0.314 & $152(140-164)$ & 0.361 & 122 (112-133) & 0.862 & 174 & $3.40(3.24-3.57)$ & 0.930 \\
\hline & & GG & 263 & $240(225-255)$ & & $143(134-153)$ & & 119 (111-128) & & 263 & $3.38(3.25-3.52)$ & \\
\hline
\end{tabular}

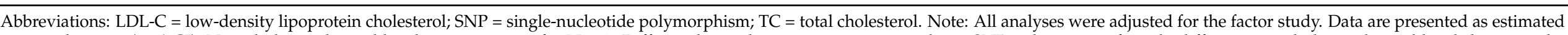

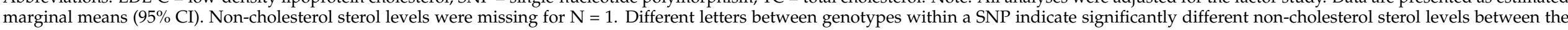

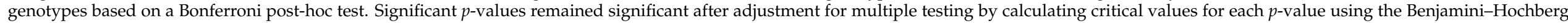
principle. ${ }^{+}$Indicates a tag SNP. ${ }^{\#}$ Recessive models are presented in the supplemental material (Figure S5). ${ }^{\$}$ Additive models are presented in the supplemental material (Table S7).

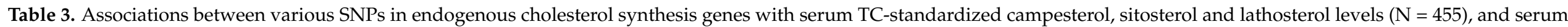
LDL-C concentrations $(\mathrm{N}=456)$.

\begin{tabular}{|c|c|c|c|c|c|c|c|c|c|c|c|c|}
\hline \multirow[t]{2}{*}{ Gene } & \multirow[t]{2}{*}{ SNP } & \multirow[t]{2}{*}{ Genotype } & \multirow[t]{2}{*}{$\mathbf{N}$} & \multicolumn{2}{|c|}{$\begin{array}{c}\text { Campesterol } \\
10^{2} \times \mu \mathrm{mol} / \mathrm{mmol} \mathrm{TC}\end{array}$} & \multicolumn{2}{|c|}{$\begin{array}{c}\text { Sitosterol } \\
10^{2} \times \mu \mathrm{mol} / \mathrm{mmol} \mathrm{TC}\end{array}$} & \multicolumn{2}{|c|}{$\begin{array}{c}\text { Lathosterol } \\
10^{2} \times \mu \mathrm{mol} / \mathrm{mmol} \mathrm{TC}\end{array}$} & \multirow[t]{2}{*}{$\mathbf{N}$} & \multicolumn{2}{|c|}{$\begin{array}{l}\mathrm{LDL}-\mathrm{C} \\
\mathrm{mmol} / \mathrm{L}\end{array}$} \\
\hline & & & & Mean $(95 \%$ CI) & $p$-Value & Mean $(95 \%$ CI) & $p$-Value & Mean $(95 \%$ CI) & $p$-Value & & Mean $(95 \%$ CI) & $p$-Value \\
\hline \multirow[t]{3}{*}{ CYP51A1 } & rs35968894 & AA & 161 & $240(222-258)$ & & $142(131-154)$ & & $115(104-124)$ & & 161 & $3.40(3.24-3.56)$ & \\
\hline & & AG & 223 & $241(224-258)$ & 0.239 & $146(135-157)$ & 0.334 & $127(118-136)$ & 0.066 & 224 & $3.38(3.23-3.53)$ & 0.976 \\
\hline & & GG & 71 & $262(238-287)$ & & $156(140-172)$ & & 117 (103-131) & & 71 & $3.39(3.17-3.60)$ & \\
\hline
\end{tabular}


Table 3. Cont.

\begin{tabular}{|c|c|c|c|c|c|c|c|c|c|c|c|c|}
\hline \multirow[t]{2}{*}{ Gene } & \multirow[t]{2}{*}{ SNP } & \multirow[t]{2}{*}{ Genotype } & \multirow[t]{2}{*}{$\mathbf{N}$} & \multicolumn{2}{|c|}{$\begin{array}{c}\text { Campesterol } \\
10^{2} \times \mu \mathrm{mol} / \mathrm{mmol} \mathrm{TC}\end{array}$} & \multicolumn{2}{|c|}{$\begin{array}{c}\text { Sitosterol } \\
10^{2} \times \mu \mathrm{mol} / \mathrm{mmol} \mathrm{TC}\end{array}$} & \multicolumn{2}{|c|}{$\begin{array}{c}\text { Lathosterol } \\
10^{2} \times \mu \mathrm{mol} / \mathrm{mmol} \mathrm{TC}\end{array}$} & \multirow[t]{2}{*}{$\mathbf{N}$} & \multicolumn{2}{|c|}{$\begin{array}{c}\text { LDL-C } \\
\mathrm{mmol} / \mathrm{L}\end{array}$} \\
\hline & & & & Mean $(95 \% \mathrm{CI})$ & $p$-Value & Mean $(95 \% \mathrm{CI})$ & $p$-Value & Mean $(95 \% \mathrm{CI})$ & $p$-Value & & Mean $(95 \% \mathrm{CI})$ & $p$-Value \\
\hline \multirow[t]{6}{*}{ DHCR24 } & rs6676774+ & AA & 75 & $231(207-256)$ & & $144(128-160)$ & & $120(106-134)$ & & 75 & $3.42(3.20-3.63)$ & \\
\hline & & $\mathrm{AG}$ & 208 & $246(230-263)$ & 0.436 & 146 (135-157) & 0.887 & $123(114-132)$ & 0.535 & 208 & $3.30(3.16-3.45)$ & 0.122 \\
\hline & & GG & 172 & $249(230-267)$ & & $148(136-160)$ & & $117(107-127)$ & & 173 & $3.48(3.33-3.64)$ & \\
\hline & rs718265 & AA & 43 & $231(200-263)$ & & $143(123-164)$ & & $117(98-134)$ & & 43 & $3.35(3.07-3.62)$ & \\
\hline & & $A G$ & 190 & $252(235-269)$ & 0.292 & $149(138-160)$ & 0.794 & $123(114-133)$ & 0.570 & 190 & $3.34(3.19-3.49)$ & 0.460 \\
\hline & & GG & 222 & $240(223-257)$ & & 145 (134-156) & & 118 (109-127) & & 223 & $3.44(3.29-3.59)$ & \\
\hline \multirow[t]{3}{*}{ HMGCR } & rs12916 ${ }^{+}$ & $\mathrm{TT}$ & 151 & $240(221-260)$ & & $145(133-158)$ & & $122(112-133)$ & & 152 & $3.22(3.05-3.39)^{\mathrm{A}}$ & \\
\hline & & $\mathrm{TC}$ & 231 & $242(226-259)$ & 0.373 & 145 (134-155) & 0.541 & 119 (110-128) & 0.838 & 231 & $3.49(3.35-3.63)^{\mathrm{B}}$ & $0.011^{@}$ \\
\hline & & $\mathrm{CC}$ & 73 & $259(234-284)$ & & $154(138-170)$ & & $122(108-135)$ & & 73 & $3.35(3.13-3.56)$ & \\
\hline \multirow[t]{3}{*}{ HSD17B7 } & rs77482353 & AA & 156 & 241 (222-259) & & $142(130-154)$ & & $121(111-131)$ & & 156 & $3.40(3.24-3.56)$ & \\
\hline & & $A G$ & 227 & $250(233-266)$ & 0.676 & $150(139-160)$ & 0.516 & $120(111-130)$ & 0.889 & 228 & $3.32(3.18-3.47)$ & 0.070 \\
\hline & & GG & 68 & $246(220-272)$ & & $150(133-167)$ & & $117(103-132)$ & & 68 & $3.60(3.72-3.83)$ & \\
\hline \multirow[t]{5}{*}{$L B R$} & rs6678087 & TT & 141 & $247(228-267)$ & & $147(134-160)$ & & $120(109-131)$ & & 141 & $3.41(3.24-3.58)$ & \\
\hline & & $\mathrm{TC}$ & 223 & $248(232-265)$ & 0.367 & 147 (136-157) & 0.988 & $121(112-130)$ & 0.997 & 223 & $3.39(2.25-3.53)$ & 0.970 \\
\hline & rs12141732 & $\mathrm{TT}$ & 226 & $241(224-258)$ & & $144(133-155)$ & & $121(111-130)$ & & 227 & $3.50(3.35-3.65) \mathrm{A}$ & \\
\hline & & $\mathrm{TC}$ & 194 & $248(232-265)$ & 0.706 & 147 (136-158) & 0.453 & $121(112-130)$ & 0.799 & 194 & $3.28(3.13-3.43)^{\text {В }}$ & $0.027^{@}$ \\
\hline & & $\mathrm{CC}$ & 34 & $251(216-286)$ & & 159 (136-182) & & $114(95-134)$ & & 34 & $3.50(3.20-3.81)$ & \\
\hline \multirow[t]{3}{*}{ MSMO1 } & rs17046216 & AA & 53 & 237 (209-266) & & $147(128-165)$ & & $113(97-128)$ & & 53 & $3.63(3.38-3.88)$ & \\
\hline & & $A G$ & 205 & $236(219-253)$ & 0.112 & 142 (131-153) & 0.347 & $121(112-131)$ & 0.542 & 206 & $3.35(3.21-3.50)$ & 0.101 \\
\hline & & GG & 197 & $256(239-273)$ & & $151(140-162)$ & & $122(112-131)$ & & 197 & $3.36(3.21-3.51)$ & \\
\hline
\end{tabular}

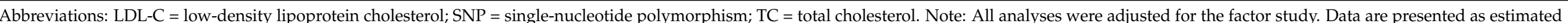

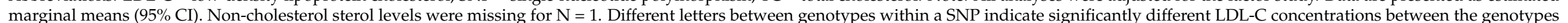

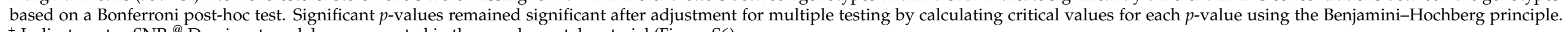
Indicates a tag SNP. ${ }^{\circledR}$ Dominant models are presented in the supplemental material (Figure S6). 


\section{Discussion}

Large inter-individual variation in intestinal cholesterol absorption and endogenous cholesterol synthesis exists, which may relate to differences in genetic background. Indeed, we found that SNPs in $A B C G 5$ and $A B C G 8$ were associated with intestinal cholesterol absorption, while SNPs in NPC1L1 were significantly associated with endogenous cholesterol synthesis. However, none of the SNPs that were associated with intestinal cholesterol absorption or endogenous synthesis were associated with serum LDL-C concentrations, whereas SNPs in HMGCR and $L B R$ did show such a relation. No associations were found for SNPs in CYP51A1, DHCR24, HSD17B7, and MSMO1 with either one of the evaluated parameters.

ABCG5 (rs4245786) was significantly related with TC-standardized serum sitosterol levels, a marker for intestinal cholesterol absorption. To the best of our knowledge, this association has not been reported before. ABCG8 (rs4245791) had tagged rs6544713 and rs4299376, which all showed significant associations with intestinal cholesterol absorption markers. A previous study in a European cohort has also reported that SNPs in ABCG8 were associated with cholesterol absorption [19]. In that study, the minor allele of rs41360247 was negatively related to cholesterol absorption and the minor allele of rs4245791 positively [19], which is in agreement with our findings.

For genes encoding enzymes of the endogenous cholesterol synthesis pathways, no significant associations with TC-standardized serum lathosterol levels were reported. Lathosterol is an intermediate in the Kandutsch-Russell pathway. To what extent the selected SNPs that are essential in endogenous cholesterol synthesis are associated with cholesterol synthesis rates in the Bloch pathway is not clear. For this, serum desmosterol should have been measured, which is specific for the Bloch pathway, whereas we analyzed lathosterol which is only part of the Kandutsch-Russell pathway. An explanation for the non-significant relations for the SNPs in the endogenous cholesterol synthesis genes that were selected in our study may be that other SNPs in these genes are associated with endogenous cholesterol synthesis, which were not included in the present study. Another explanation might be that the regulation of endogenous cholesterol synthesis is more complex and does not relate to one single SNP, as many enzymes are involved in the endogenous cholesterol synthesis pathway. In contrast to the absence of an association with lathosterol levels, SNPs in LBR (rs12141732) and HMGCR (rs12916) were significantly related with serum LDL-C concentrations. HMGCR (rs12916) was selected as tag SNP for HMGCR (rs12654264, rs3846662, and rs3846663), which also showed significant associations with serum LDL-C concentrations. For HMGCR (rs12654264, rs3846662, rs3846663, and rs12916) these associations with LDL-C concentrations agree with previous studies in Asian and European populations [38-42]. Although intestinal cholesterol absorption and endogenous cholesterol synthesis play a key role in the regulation of plasma LDL-C concentrations [2], they do not explain the significant associations between SNP in HMGCR and $L B R$ with serum LDL-C concentrations. It is likely that other genes that are involved in cholesterol homeostasis have contributed to these findings.

Interestingly, SNPs in genes involved in intestinal cholesterol absorption were not exclusively associated with markers for their postulated physiological process. However, the cholesterol absorption genes $A B C G 5, A B C G 8$, and NPC1L1 are not only expressed in the human intestine, but also in the liver $[43,44]$. On hepatocytes, $A B C G 5 / G 8$ regulates the secretion of cholesterol into bile and NPC1L1 facilitates hepatic cholesterol re-uptake, thereby finetuning an otherwise potentially large biliary and fecal loss of cholesterol [45]. In transgenic mice, overexpression of human $A B C G 5$ and $A B C G 8$ in the liver and small intestine reduced plasma plant sterol levels and fractional cholesterol absorption as measured by the fecal dual-isotope radio method [46]. In contrast, plasma lathosterol and liver mRNA levels of HMGCR were increased. Additionally, in vivo cholesterol synthesis was increased in the liver, possibly to compensate for the elevated biliary cholesterol secretion rates in these transgenic mice [46]. This animal study thus shows that ABCG5 and ABCG8 expression influences endogenous cholesterol synthesis which confirms our observations. 
Moreover, in our cohort, we noticed a similar association for an absorption gene, i.e., two SNPs in NPC1L1 (rs217429 and rs217416) were associated with endogenous cholesterol synthesis. The question remains whether these associations between SNPs in intestinal cholesterol absorption genes and lathosterol only show the reciprocal phenomenon or should also be interpreted as a possible direct effect of the SNP on hepatic cholesterol synthesis. Temel et al. have shown that hepatic NPC1L1 expression in transgenic mice increased hepatic cholesterol levels by enhancing the reuptake of cholesterol from the bile [47]. It may be that SNPs in NPC1L1 have increased the expression or activity of NPC1L1 in the liver, which in turn impacts serum lathosterol levels. Furthermore, the SNPs in $A B C G 5$ and $A B C G 8$ that showed an association with intestinal cholesterol absorption were not associated with serum LDL-C concentrations and also did not show an inverse association with endogenous cholesterol synthesis. This may suggest that the cholesterol has been eliminated from the body, via for example hepatobiliary cholesterol excretion involving ABCG5/G8 or transintestinal cholesterol efflux [2,48].

There are some points that should be considered while interpreting our data. Firstly, it should be noted that almost all selected SNPs were located in intron regions. In general, SNPs in introns do not induce changes in protein-coding sequences, suggesting that they are potentially of less functional relevance than SNPs located in exons. However, SNPs in the intron regions can impact the protein via alternative regulation of splicing [49]. This can lead to incorrectly spliced mRNA, which may ultimately affect mRNA translation and result in non-functional proteins and can also have clinical consequences [50]. SNPs in introns could also serve as markers for other functionally relevant SNPs, as should be indicated by high LD between the SNPs. Secondly, significant differences were found between all baseline characteristics, except for gender distribution, between the five different studies. This heterogeneity between study populations was taken into account by correcting for the factor study in our analyses. In addition, only European individuals were included, which has further minimized this heterogeneity. In four studies, only individuals with a stable body weight (weight gain or loss of $<3 \mathrm{~kg}$ for studies 1,2 and 3 and $<2 \mathrm{~kg}$ for study 5) could participate. For study 4 , a stable body weight was not an inclusion criterion. It is therefore possible that some of the participants lost or gained some weight in the months preceding the study. However, it is not expected that possible changes in weight were related to a specific genotype group and therefore biased the results. Thirdly, this study had a relatively small sample size. This suggests that the significant findings that we found reflect strong associations. Our results can therefore help to determine whether individuals with specific genotypes are more sensitive to specific nutritional and pharmacological interventions, such as foods enriched with plant sterols or stanols, or ezetimibe and statin treatment. To illustrate, 4-week statin treatment in women with familiar hypercholesterolemia resulted in a significantly smaller percentage reduction in LDL-C concentrations in women with the AA genotype of HMGCR (rs3846662) compared to women with the other genotypes. Moreover, statin efficacy was significantly decreased in the AA group compared with women with the other genotypes [51]. This suggests that genotyping SNPs, even those located in the intron region, may play an important role in the development of more personalized treatment. Finally, an independent cohort in which we could replicate the positive findings was unavailable. Therefore, an additional study is needed to reach greater validity.

\section{Conclusions}

This study showed that several SNPs in genes that are essential in intestinal cholesterol absorption were associated with serum markers for intestinal cholesterol absorption and/or endogenous cholesterol synthesis. In addition, a number of SNPs in genes that are essential in endogenous cholesterol synthesis were associated with serum LDL-C concentrations in a European cohort.

Supplementary Materials: The following are available online at https:/ /www.mdpi.com/article/10.3 390/biomedicines9101475/s1, Figure S1: Schematic overview of the intestinal cholesterol absorption 
pathway, Figure S2: The endogenous cholesterol synthesis pathway, Table S1: List of full names of genes included in the present study, Table S2: Information given by the Precision Medicine Research Array for the two SNPs in ABCG8 with an unknown rs-number, Table S3: Baseline characteristics for all participants and stratified by study, Table S4: Associations between intestinal cholesterol absorption markers, an endogenous cholesterol synthesis marker and serum LDL-C concentrations, Table S5: The location and allele frequencies for various SNPs in intestinal cholesterol absorption and endogenous cholesterol synthesis genes for 456 participants, Figure S3: Pairwise LD among SNPs in ABCG5 and NPC1L1, Figure S4: Pairwise LD among SNPs in MSMO1, DHCR7, DHCR24, and LBR, Table S6: Associations between various SNPs in cholesterol absorption genes, that were either captured by a tag SNP or contained a genotype group $<12$ individuals, with serum TC-standardized campesterol, sitosterol and lathosterol levels $(\mathrm{N}=455)$, and serum LDL-C concentrations $(\mathrm{N}=456)$, Figure S5: Association between SNPs NPC1L1 (rs127429) and NPC1L1 (rs217416) with serum levels of cholesterol-standardized lathosterol using recessive models, Table S7: Associations between SNPs in intestinal cholesterol absorption genes with TC-standardized non-cholesterol sterols using additive models $(\mathrm{N}=455)$, Table S8: Associations between various SNPs in genes involved in intestinal cholesterol absorption with serum total cholesterol concentrations $(\mathrm{N}=456)$, Figure S6: Association between SNPs HMGCR (rs12916) and LBR (rs12141732) with serum LDL-C concentrations using dominant models, Table S9: Associations between various SNPs in endogenous cholesterol synthesis genes, that were either captured by a tag SNP or contained a genotype group $<12$ individuals, with serum TC-standardized campesterol, sitosterol and lathosterol levels $(\mathrm{N}=455)$, and serum LDL-C concentrations $(\mathrm{N}=456)$, Table S10: Associations between various SNPs in genes involved in endogenous cholesterol synthesis with serum total cholesterol concentrations $(\mathrm{N}=456)$.

Author Contributions: Conceptualization, F.B.A.M., M.M.S., J.P. and R.P.M.; methodology, F.B.A.M. and M.M.S.; formal analysis, F.B.A.M. and M.M.S.; writing-original draft preparation, F.B.A.M. and M.M.S.; writing-review and editing, J.P. and R.P.M.; supervision, J.P. and R.P.M. All authors have read and agreed to the published version of the manuscript.

Funding: This research received no external funding.

Institutional Review Board Statement: The studies were conducted according to the guidelines of the Declaration of Helsinki, and approved by the Medical Ethics Committee of Maastricht University (Study 1: MEC 96-181, approval date: 20 December 1996; Study 2: MEC 97-204, approval date: 11 March 1998; Study 3: MEC 99-206, approval date: 6 December 1999; Study 4: MEC 07-3-027, approval date: 13 April 2007; Study 5: METC 12-3-005, approval date: 14 March 2012).

Informed Consent Statement: Informed consent was obtained from all subjects involved in the study.

Data Availability Statement: All data are included in this manuscript.

Conflicts of Interest: The authors declare no conflict of interest.

\section{References}

1. Mc Auley, M.T.; Wilkinson, D.J.; Jones, J.J.L.; Kirkwood, T.B.L. A whole-body mathematical model of cholesterol metabolism and its age-associated dysregulation. BMC Syst. Biol. 2012, 6, 130. [CrossRef]

2. Luo, J.; Yang, H.; Song, B.L. Mechanisms and regulation of cholesterol homeostasis. Nat. Rev. Mol. Cell Biol. 2020, 21, 225-245. [CrossRef] [PubMed]

3. Jia, L.; Betters, J.L.; Yu, L. Niemann-pick C1-like 1 (NPC1L1) protein in intestinal and hepatic cholesterol transport. Annu. Rev. Physiol. 2011, 73, 239-259. [CrossRef] [PubMed]

4. Jakulj, L.; Vissers, M.N.; Tanck, M.W.T.; Hutten, B.A.; Stellaard, F.; Kastelein, J.J.P.; Dallinga-Thie, G.M. ABCG5/G8 polymorphisms and markers of cholesterol metabolism: Systematic review and meta-analysis. J. Lipid Res. 2010, 51, 3016-3023. [CrossRef] [PubMed]

5. Bloch, K. The biological synthesis of cholesterol. Science 1965, 150, 19-28. [CrossRef] [PubMed]

6. Kandutsch, A.A.; Russell, A.E. Preputial gland tumor sterols. I. The occurrence of 24,25-dihydrolanosterol and a comparison with liver and the normal gland. J. Biol. Chem. 1959, 234, 2037-2042. [CrossRef]

7. Kandutsch, A.A.; Russell, A.E. Preputial gland tumor sterols. 2. The identification of 4 alpha-methyl-Delta 8-cholesten-3 beta-ol. J. Biol. Chem. 1960, 235, 2253-2255. [CrossRef]

8. Kandutsch, A.A.; Russell, A.E. Preputial gland tumor sterols. 3. A metabolic pathway from lanosterol to cholesterol. J. Biol. Chem. 1960, 235, 2256-2261. [CrossRef]

9. Miettinen, T.A.; Tilvis, R.S.; Kesaniemi, Y.A. Serum plant sterols and cholesterol precursors reflect cholesterol absorption and synthesis in volunteers of a randomly selected male population. Am. J. Epidemiol. 1990, 131, 20-31. [CrossRef] 
10. Santosa, S.; Varady, K.A.; AbuMweis, S.; Jones, P.J.H. Physiological and therapeutic factors affecting cholesterol metabolism: Does a reciprocal relationship between cholesterol absorption and synthesis really exist? Life Sci. 2007, 80, 505-514. [CrossRef]

11. Miettinen, T.A.; Gylling, H. Synthesis and absorption markers of cholesterol in serum and lipoproteins during a large dose of statin treatment. Eur. J. Clin. investig. 2003, 33, 976-982. [CrossRef]

12. Naruse, R.; Hori, K.; Terasawa, T.; Hara, K.; Suetsugu, M.; Takebayashi, K.; Morita, K.; Aso, Y.; Inukai, T. Alterations of plant sterols, lathosterol, oxidative stress and inflammatory markers after the combination therapy of ezetimibe and statin drugs in type 2 diabetic patients. Obes. Res. Clin. Pract. 2015, 9, 67-74. [CrossRef]

13. Bosner, M.S.; Lange, L.G.; Stenson, W.F.; Ostlund, R.E., Jr. Percent cholesterol absorption in normal women and men quantified with dual stable isotopic tracers and negative ion mass spectrometry. J. Lipid Res. 1999, 40, 302-308. [CrossRef]

14. Wu, A.H.; Ruan, W.; Todd, J.; Lynch, K.L. Biological variation of beta-sitosterol, campesterol, and lathosterol as cholesterol absorption and synthesis biomarkers. Clin. Chim. Acta 2014, 430, 43-47. [CrossRef] [PubMed]

15. Alphonse, P.A.; Jones, P.J. Revisiting Human Cholesterol Synthesis and Absorption: The Reciprocity Paradigm and its Key Regulators. Lipids 2016, 51, 519-536. [CrossRef] [PubMed]

16. Renner, O.; Lütjohann, D.; Richter, D.; Strohmeyer, A.; Schimmel, S.; Müller, O.; Stange, E.F.; Harsch, S. Role of the ABCG8 19H risk allele in cholesterol absorption and gallstone disease. BMC Gastroenterol. 2013, 13, 30. [CrossRef] [PubMed]

17. Berge, K.E.; von Bergmann, K.; Lutjohann, D.; Guerra, R.; Grundy, S.M.; Hobbs, H.H.; Cohen, J.C. Heritability of plasma noncholesterol sterols and relationship to DNA sequence polymorphism in ABCG5 and ABCG8. J. Lipid Res. 2002, 43, 486-494. [CrossRef]

18. Wolff, E.; Vergnes, M.-F.; Defoort, C.; Planells, R.; Portugal, H.; Nicolay, A.; Lairon, D. Cholesterol absorption status and fasting plasma cholesterol are modulated by the microsomal triacylglycerol transfer protein $-493 \mathrm{G} / \mathrm{T}$ polymorphism and the usual diet in women. Genes. Nutr. 2011, 6, 71-79. [CrossRef]

19. Teupser, D.; Baber, R.; Ceglarek, U.; Scholz, M.; Illig, T.; Gieger, C.; Holdt, L.M.; Leichtle, A.; Greiser, K.H.; Huster, D.; et al. Genetic regulation of serum phytosterol levels and risk of coronary artery disease. Circ. Cardiovasc. Genet. 2010, 3, $331-339$. [CrossRef]

20. Chung, J.Y.; Cho, S.K.; Oh, E.S.; Lee, D.H.; Lim, L.A.; Jang, S.B.; Lee, Y.J.; Park, K.; Park, M.S. Effect of HMGCR variant alleles on low-density lipoprotein cholesterol-lowering response to atorvastatin in healthy Korean subjects. J. Clin. Pharmacol. 2012, 52, 339-346. [CrossRef]

21. Chasman, D.I.; Posada, D.; Subrahmanyan, L.; Cook, N.R.; Stanton, V.P., Jr.; Ridker, P.M. Pharmacogenetic study of statin therapy and cholesterol reduction. JAMA 2004, 291, 2821-2827. [CrossRef]

22. Krauss, R.M.; Mangravite, L.M.; Smith, J.D.; Medina, M.W.; Wang, D.; Guo, X.; Rieder, M.J.; Simon, J.A.; Hulley, S.B.; Waters, D.; et al. Variation in the 3-hydroxyl-3-methylglutaryl coenzyme a reductase gene is associated with racial differences in low-density lipoprotein cholesterol response to simvastatin treatment. Circulation 2008, 117, 1537-1544. [CrossRef] [PubMed]

23. Hegele, R.A.; Guy, J.; Ban, M.R.; Wang, J. NPC1L1 haplotype is associated with inter-individual variation in plasma low-density lipoprotein response to ezetimibe. Lipids Health Dis. 2005, 4, 16. [CrossRef] [PubMed]

24. Abdullah, M.M.; Cyr, A.; Lepine, M.C.; Eck, P.K.; Couture, P.; Lamarche, B.; Jones, P.J. Common Variants in Cholesterol Synthesisand Transport-Related Genes Associate with Circulating Cholesterol Responses to Intakes of Conventional Dairy Products in Healthy Individuals. J. Nutr. 2016, 146, 1008-1016. [CrossRef] [PubMed]

25. Herron, K.L.; McGrane, M.M.; Waters, D.; Lofgren, I.E.; Clark, R.M.; Ordovas, J.M.; Fernandez, M.L. The ABCG5 polymorphism contributes to individual responses to dietary cholesterol and carotenoids in eggs. J. Nutr. 2006, 136, 1161-1165. [CrossRef] [PubMed]

26. World Health Organization. Body Mass Index-BMI. Available online: https://www.euro.who.int/en/health-topics/diseaseprevention/nutrition/a-healthy-lifestyle/body-mass-index-bmi (accessed on 4 October 2021).

27. Kerckhoffs, D.A.; Hornstra, G.; Mensink, R.P. Cholesterol-lowering effect of $\beta$-glucan from oat bran in mildly hypercholesterolemic subjects may decrease when $\beta$-glucan is incorporated into bread and cookies. Am. J. Clin. Nutr. 2003, 78, 221-227. [CrossRef] [PubMed]

28. Plat, J.; Mensink, R.P. Vegetable oil based versus wood based stanol ester mixtures: Effects on serum lipids and hemostatic factors in non-hypercholesterolemic subjects. Atherosclerosis 2000, 148, 101-112. [CrossRef]

29. Plat, J.; van Onselen, E.N.M.; van Heugten, M.M.A.; Mensink, R.P. Effects on serum lipids, lipoproteins and fat soluble antioxidant concentrations of consumption frequency of margarines and shortenings enriched with plant stanol esters. Eur. J. Clin. Nutr. 2000, 54, 671-677. [CrossRef]

30. De Smet, E.; Mensink, R.P.; Lütjohann, D.; Plat, J. Acute effects of plant stanol esters on postprandial metabolism and its relation with changes in serum lipids after chronic intake. Eur. J. Clin. Nutr. 2015, 69, 127-133. [CrossRef]

31. Friedewald, W.T.; Levy, R.I.; Fredrickson, D.S. Estimation of the concentration of low-density lipoprotein cholesterol in plasma, without use of the preparative ultracentrifuge. Clin. Chem. 1972, 18, 499-502. [CrossRef]

32. Mackay, D.S.; Jones, P.J.; Myrie, S.B.; Plat, J.; Lutjohann, D. Methodological considerations for the harmonization of non-cholesterol sterol bio-analysis. J. Chromatogr. Biomed Analyt. Technol. Biomed. Life Sci. 2014, 957, 116-122. [CrossRef]

33. Axiom ${ }^{\mathrm{TM}}$ Precision Medicine Research Array (PMRA) Applied Biosystems ${ }^{\mathrm{TM}}$, Catalog Number: 902981. Available online: https:/ / www.thermofisher.com/order/catalog/product/902981\#/902981 (accessed on 20 January 2021). 
34. Purcell, S.; Chang, C. PLINK, Version: 1.90 Beta. Available online: www.cog-genomics.org/plink/1.9/ (accessed on 20 January 2021).

35. Barrett, J.C.; Fry, B.; Maller, J.; Daly, M.J. Haploview: Analysis and visualization of LD and haplotype maps. Bioinformatics 2005, 21, 263-265. [CrossRef]

36. Gabriel, S.B.; Schaffner, S.F.; Nguyen, H.; Moore, J.M.; Roy, J.; Blumenstiel, B.; Higgins, J.; DeFelice, M.; Lochner, A.; Faggart, M.; et al. The structure of haplotype blocks in the human genome. Science 2002, 296, 2225-2229. [CrossRef]

37. Database of Single Nucleotide Polymorphisms (dbSNP). Bethesda (MD): National Center for Biotechnology Information, National Library of Medicine. dbSNP Accession:(dbSNP Build ID: 154). Available online: http://www.ncbi.nlm.nih.gov/SNP/ (accessed on 28 January 2021).

38. Burkhardt, R.; Kenny, E.E.; Lowe, J.K.; Birkeland, A.; Josowitz, R.; Noel, M.; Salit, J.; Maller, J.B.; Pe'er, I.; Daly, M.J.; et al. Common SNPs in HMGCR in micronesians and whites associated with LDL-cholesterol levels affect alternative splicing of exon13. Arterioscler. Thromb. Vasc. Biol. 2008, 28, 2078-2084. [CrossRef]

39. Kathiresan, S.; Melander, O.; Anevski, D.; Guiducci, C.; Burtt, N.P.; Roos, C.; Hirschhorn, J.N.; Berglund, G.; Hedblad, B.; Groop, L.; et al. Polymorphisms associated with cholesterol and risk of cardiovascular events. N. Engl. J. Med. 2008, 358, 1240-1249. [CrossRef]

40. Kathiresan, S.; Melander, O.; Guiducci, C.; Surti, A.; Burtt, N.P.; Rieder, M.J.; Cooper, G.M.; Roos, C.; Voight, B.F.; Havulinna, A.S.; et al. Six new loci associated with blood low-density lipoprotein cholesterol, high-density lipoprotein cholesterol or triglycerides in humans. Nat. Genet. 2008, 40, 189-197. [CrossRef] [PubMed]

41. Wurtz, P.; Wang, Q.; Soininen, P.; Kangas, A.J.; Fatemifar, G.; Tynkkynen, T.; Tiainen, M.; Perola, M.; Tillin, T.; Hughes, A.D.; et al. Metabolomic Profiling of Statin Use and Genetic Inhibition of HMG-CoA Reductase. J. Am. Coll. Cardiol. 2016, 67, 1200-1210. [CrossRef] [PubMed]

42. Angelini, S.; Rosticci, M.; Massimo, G.; Musti, M.; Ravegnini, G.; Consolini, N.; Sammarini, G.; D’Addato, S.; Rizzoli, E.; Botbayev, D.; et al. Relationship between Lipid Phenotypes, Overweight, Lipid Lowering Drug Response and KIF6 and HMG-CoA Genotypes in a Subset of the Brisighella Heart Study Population. Int. J. Mol. Sci 2018, 19, 49. [CrossRef] [PubMed]

43. Repa, J.J.; Berge, K.E.; Pomajzl, C.; Richardson, J.A.; Hobbs, H.; Mangelsdorf, D.J. Regulation of ATP-binding Cassette Sterol Transporters ABCG5 and ABCG8 by the Liver X Receptors $\alpha$ and $\beta$. J. Biol. Chem. 2002, 277, 18793-18800. [CrossRef]

44. Klett, E.L.; Patel, S.B. Biomedicine. Will the real cholesterol transporter please stand up. Science 2004, 303, 1149-1150. [CrossRef] [PubMed]

45. Brown, J.M.; Yu, L. Opposing Gatekeepers of Apical Sterol Transport: Niemann-Pick C1-Like 1 (NPC1L1) and ATP-Binding Cassette Transporters G5 and G8 (ABCG5/ABCG8). Immunol. Endocr. Metab. Agents. Med. Chem. 2009, 9, 18-29. [CrossRef] [PubMed]

46. Yu, L.; Li-Hawkins, J.; Hammer, R.E.; Berge, K.E.; Horton, J.D.; Cohen, J.C.; Hobbs, H.H. Overexpression of $A B C G 5$ and $A B C G 8$ promotes biliary cholesterol secretion and reduces fractional absorption of dietary cholesterol. J. Clin. Invest. 2002, 110, 671-680. [CrossRef] [PubMed]

47. Temel, R.E.; Tang, W.; Ma, Y.; Rudel, L.L.; Willingham, M.C.; Ioannou, Y.A.; Davies, J.P.; Nilsson, L.M.; Yu, L. Hepatic NiemannPick C1-like 1 regulates biliary cholesterol concentration and is a target of ezetimibe. J. Clin. Invest. 2007, 117, 1968-1978. [CrossRef] [PubMed]

48. van der Velde, A.E.; Brufau, G.; Groen, A.K. Transintestinal cholesterol efflux. Curr. Opin. Lipidol. 2010, 21, 167-171. [CrossRef] [PubMed]

49. Jo, B.S.; Choi, S.S. Introns: The Functional Benefits of Introns in Genomes. Genomics. Inform. 2015, 13, 112-118. [CrossRef]

50. Wang, G.S.; Cooper, T.A. Splicing in disease: Disruption of the splicing code and the decoding machinery. Nat. Rev. Genet. 2007, 8, 749-761. [CrossRef]

51. Leduc, V.; Bourque, L.; Poirier, J.; Dufour, R. Role of rs3846662 and HMGCR alternative splicing in statin efficacy and baseline lipid levels in familial hypercholesterolemia. Pharm. Genom. 2016, 26, 1-11. [CrossRef] 